\title{
Quantum Theory and Symmetries
}


This page is intentionally left blank 
Proceedings of the Second International Symposium on

\section{Quantum Theory and Symmetries}

Kraków, Poland

July $18-21,2001$

Editors

Edward Kapuścik

\section{Andrzej Horzela}

H. Niewodniczański Institute of Nuclear Physics Kraków, Poland 


\section{Published by}

World Scientific Publishing Co. Pte. Ltd.

P O Box 128, Farrer Road, Singapore 912805

USA office: Suite 1B, 1060 Main Street, River Edge, NJ 07661

UK office: 57 Shelton Street, Covent Garden, London WC2H 9HE

\section{British Library Cataloguing-in-Publication Data}

A catalogue record for this book is available from the British Library.

\section{QUANTUM THEORY AND SYMMETRIES}

Proceedings of the Second International Symposium

Copyright $\odot 2002$ by World Scientific Publishing Co. Pte. Ltd.

All rights reserved. This book, or parts thereof, may not be reproduced in any form or by any means, electronic or mechanical, including photocopying, recording or any information storage and retrieval system now known or to be invented, without written permission from the Publisher.

For photocopying of material in this volume, please pay a copying fee through the Copyright Clearance Center, Inc., 222 Rosewood Drive, Danvers, MA 01923, USA. In this case permission to photocopy is not required from the publisher.

ISBN $981-02-4887-3$ 


\section{PREFACE}

It is our great pleasure to present to the Physics community the Proceedings of the Second International Symposium Quantum Theory and Symmetries held at the Henryk Niewodniczański Institute of Nuclear Physics in Kraków from 18 till 21 July 2001. The Kraków Symposium was the second one in a series of bi-annual symposia presided over by the International Board consisting of: H.-D. Doebner (Chairman), S.-T. Ali, L. L. Boyle, V. S. Dobrev, E. Kapuścik, V. I. Man'ko, F. Mansouri, G. Marmo, M. A. del Olmo, G. S. Pogosyan, M. Rasetti, T. H. Seligman and A. I. Solomon. The first symposium of the series took place in Goslar (Germany) in 1999 and its proceedings were published by World Scientific in 2000 .

The members of the Advisory Committee of the Kraków symposium were: A. Bohm, C. Burdik, Z. Haba, G. C. Hegerfeldt, A. Jamiołkowski, R. Kerner, Y. S. Kim, A. U. Klimyk, A. Kossakowski, M. A. Mańko, B. Mielnik, G. Marmo, Y. Ne'eman, A. Odzijewicz, L. O'Raifeartaigh, J. Rembieliński, G. Rudolph, A. S. Shumovsky, A. N. Sissakian and I. Todorov. While preparing the conference, in November 2000, we got sad news that Professor Lochlain O'Raifeartaigh, a great man and scientist and our longtime friend, had passed away. Professors H.-D. Doebner and V. I. Man'ko paid a Memorial Tribute to him just after the Symposium opening, while a Memorial Session dedicated to him, arranged on the second day of the symposium, reminded the participants of this good and modest man whom we owe so much to and whom we badly miss.

The participants of the Symposium presented over 100 talks: 20 plenary lectures and more than 80 contributions given during parallel sessions, and covering aspects belonging to the foundations of quantum theory, quantum optics, quantum computing and control, the Lie algebraic methods, quantum groups and noncommutative geometry, gauge, field and string theories, integrable systems, symmetries in molecular physics, as well as discrete periodic and aperiodic systems. When selecting the material for the present volume, we decided to publish all contributions in alphabetical order, distingushing only between plenary lectures and contributions. In this way, any confusing overlaps between different sessions have been avoided. However, we would like to mention two associated workshops held as parallel sessions of the Symposium. These were: the 4th Workshop on Lie Theory and its Applications in Physics, organized by Professors H-D. Doebner and V. S. Dobrev, and the 3rd Workshop on Extensions of Quantum Theories, organized by one of us (A. H.). Unfortunately, some of the participants were not able to send their 
contributions in time, and thus these could not be included in this volume.

We would like to thank our sponsoring institutions whose help and financial support made the Symposium possible, namely the Polish State Commitee for Scientific Research, the Henryk Niewodniczański Institute of Nuclear Physics, the Cracow Pedagogical Academy, the College of Computer Science in Lódź, and the Joint Institute of Nuclear Research in Dubna. We are personally indepted to Professor Andrzej Budzanowski, Director General, and to Dr. Maria Pollak - Stachura, Deputy Director, both of the Henryk Niewodniczański Institute of Nuclear Physics, Professor Michał Śliwa, Rector of the Cracow Pedagogical Academy, Ms. Aniela Bednarek, Director of the College of Computer Science, and to Professor Alexey N. Sissakian, Deputy Director General of the Joint Institute of Nuclear Research. We also would like to thank all the members of the Local Organizing Committee: K. Bajan, A. Baran, A. Bieniek, P. Błasiak, W. Kantor, A. Korzeniowska, P. Łach, E. Pagaczewska, W. Polak, D. Wcisło and D. Żenczykowska, whose enthusiastic involvement contributed greatly to the success of the Symposium.

We have no doubt that all readers will find this comprehensive volume interesting, providing new insights into various problems of quantum physics and its most powerful tool, the theory of symmetries. A more extended discussion of the various related problems can be found in the Special Issue of Journal of Physics A entitled Foundations of Quantum Theory, with Professor Vladimir I. Man'ko and both of us acting as Guest Editors. To this respect let us express our gratitude to the Editorial Board of the Journal of Physics A (London, UK).

We are convinced that the idea of bi-annual meetings devoted specifically to the subject in question will be successfully continued and that all the participants of the Kraków conference will meet again as planned during the Third International Symposium on Quantum Theory and Symmetries to take place in Cincinatti (USA) in 2003.

Kraków, January 2002

Andrzej Horzela and Edward Kapuścik Symposium Organizers

H.Niewodniczański Institute of Nuclear Physics Kraków, Poland http://qts2.ifj.edu.pl 


\section{CONTENTS}

Preface

\section{Plenary Lectures}

The Interacting Fock Space of Haldane's Exclusion Statistics

L. Accardi and M. Nhani

Complex Hamiltonians Having Real Spectra

C. M. Bender

From Resonances to Poincaré Semigroups

A. R. Bohm, B. A. Tay, and P. Kielanowski

Quantum Field Theory as Dynamical System

H. J. Borchers

Beta-Lattices for Aperiodic Order

J.-P. Gazeau

Generalized Symmetries and Time

M. Heller

Integrable Hierarchies and the WDVV-equations

G. F. Helminck

Global Gauss Law for Lattice QCD

J. Kijowski and G. Rudolph

Quantum Entanglement and Symmetries

M. Kuś

From Noncommutative Space-time to Quantum Relativistic

Symmetries with Fundamental Mass Parameter

J. Lukierski

Tomographic Map within the Framework of Star-product

Quantization

O. V. Man'ko, V. I. Man'ko, and G. Marmo 
Algorithmic Cooling and Scalable Quantum Computers: Ways

to Improve the Space-time Requirements of the Algorithm

T. Mor and Y. Weinstein

Quantum Theory on the Torus with Magnetic Field

H. Narnhofer

Nonlocal Reflection by Photonic Barriers

G. Nimtz and A. Haibel

Lightfront Formalism versus Holography\&Chiral Scanning

B. Schroer

Broken Symmetries

W. Thirring

Gauge Theories on Non-commutative Spaces

J. Wess

\section{Contributions}

Algebra of Differential Forms with Exterior Differential $d^{3}=0$ in Dimension One

V. Abramov and N. Bazunova

Quantum Probability and the Non-locality Issue in Quantum Theory

L. Accardi and M. Regoli

On the Electromagnetic Reciprocity-like Theorems

G. N. Afanasiev

Intertwining Operators for Schrödinger Algebras and Hierarchy

of Invariant Equations

N. Aizawa, V. K. Dobrev, and H.-D. Doebner

Symmetries and Reduced Systems of Bethe Equations

V. A. Andreev

Construction of Wavelets Basis for the Fibonacci Chain via the Spline Functions of Various Orders

M. Andrle 
Algebraic Model of Molecular Thermodynamics

M. Angelova and A. Frank

Induced Representations of Bicrossproduct Algebras

O. Arratia and M. A. del Olmo

Contractions of the Finite Oscillator

N. M. Atakishiyev, G. S. Pogosyan, and K. B. Wolf

Separation of Discrete Variables in the 2-dim Finite Oscillator

261

N. M. Atakishiyev, G. S. Pogosyan, L. E. Vicent,

and $K$. B. Wolf

A Glimpse at Mathematical Diffraction Theory

M. Baake

Exactly Solvable Hamiltonians Connected with $s u_{q}(2)$ and

Quantum Optical Models

A. Ballesteros and S. M. Chumakov

Dynamical Coalgebra Symmetry and Integrability

A. Ballesteros, F. J. Herranz, and O. Ragnisco

$\pi \rho \omega$ Vertex in Nuclear Matter

A. Baran, A. Bieniek, and W. Broniowski

Unusual Eigenvalue Problems

291

C. M. Bender

Electroweak Interaction, Fermions and Vector Bosons from

Gauge and Space-time Symmetry Unification

J. Besprosvany

In Search of Alternatives to Higgs Fields

I. L. Bogolubsky and A. A. Bogolubskaya

q-Plane Wave Solutions of q-Maxwell Equations

V. K. Dobrev and S. T. Petrov

Symmetries and Wavelets 
On Interpretations and Constructions of Classical

Dynamical r-matrices

L. Fehér and A. Gábor

Decoupling of Translations from Homogeneous Transformations

in Inhomogeneous Quantum Groups

G. Fiore

Symmetries in the Universe at Large Scales

P. Flin, K. Bajan, and W. Godtowski

From Geometry to Quantum Computation

K. Fujii

Partition Function Zeros of Aperiodic Ising Models

U. Grimm and P. Repetowicz

Cayley-Klein Contractions of Orthosymplectic Superalgebras

N. A. Gromov, I. V. Kostyrkov, and V. V. Kuratov

Distinguished Bases of $s l(n, C)$ and their Symmetries

M. Havliček, E. Pelantová, J. Patera, and J. Tolar

Quantum Effects in Matter-Wave Diffraction

G. C. Hegerfeldt and T. Köhler

Time-type Quantum Deformation of the Conformal Algebra so $(4,2)$

F. J. Herranz

$q$-Laplace Operator Related to the Quantum Unitary Group

383

N. Z. Iorgov and A. U. Klimyk

Contraction and Interbases Expansions on N-sphere

A. A. Izmest'ev, G. S. Pogosyan, A. N. Sissakian, and $P$. Winternitz

Quantized Symmetrizations and $\star$-Products

H. P. Jakobsen

Uniqueness Results by Covariance in Covariant Quantum Mechanics J. Janyška and M. Modugno 
Waves and Solitons in Born-Infeld Electrodynamics

Quasiperiodic Patterns in Faraday Instability and

Adapted Beta-Lattice

A. D. el Kharrat, J. P. Gazeau, M. Guidy, and S. Metens

On the $S U(2)$ Wigner Function Dynamics

A. B. Klimov and S. M. Chumakov

VI Hilbert's Problem, Infinite Lie Groups and Relativistic Physics N. P. Konopleva

On the Uncertainty Relations and Squeezed States for a

Quantum Particle on a Circle

K. Kowalski and J. Rembieliński

Effective Dipole-type Field Approach and the Dual Higgs Model

G. A. Kozlov

CP Violation in B Decays: The Role of Final-state Interactions

$P$. Each and P. Żenczykowski

Spinor Algebras and Extended Superconformal Algebras

M. A. Lledó and V. S. Varadarajan

Wavelets and Tomograms in Information Processing

M. A. Man'ko

Spectral Triples for the Kronecker Foliation

R. Matthes, O. Richter and G. Rudolph

Non-commutative Space-time Geometry and Principle

of Gauge Invariance

R. M. Mir-Kasimov

An Integrable Relativistic Three Body Problem with Spin and an Application

M. Moshinsky 
Representations of $S O_{0}(p+1, q) \times_{s} R^{p+q+1}$ from its Deformations

P. Moylan

Covariance Approach to Quantum Theory

J. Naudts

Super Duffin-Kemmer-Petiau Algebra

S. Okubo

Deformed Jacobson Generators of the Algebra $U_{q}[\operatorname{sl}(n+1)]$ and their Fock Representations

T. D. Palev, N. I. Stoilova, and J. Van der Jeugt

Coherent States from Combinatorial Sequences

K. A. Penson and A. I. Solomon

Generalized Coherent States Associated with the $C_{\lambda}$-Extended

Oscillator

C. Quesne

Asymmetric Double Sinh-Gordon Theory in 1+1 Dimensions

A. Radosz, K. Ostasiewicz, P. Magnuszewski, and P. Machnikowski

Quantum Field Theory in Absence of the Positivity Condition

S. Rouhani and M. V. Takook

On the Gauge Orbit Space Stratification

G. Rudolph, M. Schmidt, and I. P. Volobuev

Group-theoretical Aspects of Control of Quantum Systems

S. G. Schirmer and A. I. Solomon

Can the Breaking of Time-reversal Symmetry Introduce

Bifurcations in Quantum Mechanics?

D. A. Schuch

Looking for a Time Operator

574

M. Skulimowski

Quantum and Classical Models Based on $G L(n)$-symmetry

J. J. Stawianowski 
$q$-boson Realization of the Positive Discrete Series of Representations of the $U_{q}(2,1)$ Quantum Algebra Yu. F. Smirnov and Yu. I. Kharitonov

Isotropic Quantized Minkowski Space and Poincaré Group K. A. Smoliński

Construction of Raising and Lowering Operators for $U_{q}(s l(2, R))$. P. Śt̆oviček

Exactly Solvable Time-dependent Potentials in Quantum Mechanics A. A. Suzko

Representations for Selected Types of Diffusion Systems R. Twarock

Quantum Mechanics at the Planck Scale F. Winterberg

Generalized PT Symmetry M. Znojil 